\title{
Lentivirus Vector CCR5 shRNA/TRIM5alpha/TAR Decoy- transduced Autologous CD34-positive Hematopoietic Progenitor Cells
}

National Cancer Institute

\section{Source}

National Cancer Institute. Lentivirus Vector CCR5 ShRNA/TRIM5alpha/TAR Decoy-

transduced Autologous CD34-positive Hematopoietic Progenitor Cells. NCI Thesaurus. Code C123931.

Autologous, CD34-positive hematopoietic progenitor cells (HPCs) transduced with a lentiviral vector encoding three anti-human immunodeficiency virus (HIV) genes: a short hairpin RNA (shRNA) that targets human chemokine receptor 5 (CCR5), a human/rhesus macaque chimeric tripartite motif-containing protein 5 alpha isoform (TRIM5alpha), and a TAT activation response (TAR) decoy, as well as a pre-selective cell-surface marker, which is a truncated and mutated form of human CD25, used to potentially provide resistance against human immunodeficiency virus (HIV) infection. Human autologous CD34-positive HPCs are isolated and transduced ex vivo with the lentiviral vector. Upon pre-selection, purification using CD25 immunomagnetic separation, and subsequent administration of effectively transduced HPCs, the HPCs display 3 separate mechanisms of action against HIV infection: CCR5 shRNA binds to CCR5 mRNA and inhibits the expression of CCR5, a HIV-1 co-receptor that mediates HIV attachment and host cell entry; TRIM5alpha prevents HIV genome integration upon cell entry; and the TAR decoy binds to the HIV TAT protein and prevents TAT-dependent viral gene transcription, thereby preventing HIV replication. Upon transfer of the lentivirus vector CCR5 shRNA/TRIM5alpha/TAR decoy-transduced autologous CD34-positive HPCs into the patient, the HPCS are resistant to HIV entry and replication and could provide long-term protection against both HIV infection and HIV-associated cancers. 